\title{
Project Management, a bit of communication helps too, on succeeding
}

\author{
E. Gentili \\ C. Nidasio, M. Varchetta \\ University of Brescia - Dipartimento di Ingegneria Meccanica \\ Via Branze, 38 - 25123 Brescia - Italy \\ $\mathrm{Tel}+39+30 / 3715578$ \\ Fax $+39+30 / 3702448$ \\ E-mailgentili@bsing.ing.unibs.it
}

\begin{abstract}
The following paper describes innovative methods and tools for planning and following up software development projects. A research study has been undertaken by a multinational company developing software applications and systems. The research study was based on an extensive literature survey in order to draw up a theoretical frame of reference and knowledge platform in the field of Project Management. The Anatomy concept joined with the On Line Project Monitoring System aim to represent a coherent proposal for project monitoring and control projects.
\end{abstract}

\section{Keywords}

Anatomy, Anatomical Chart, On Line Project Monitoring System, Project Manager (PM) 


\section{INTRODUCTION}

The research-work performed in the company consists in an evaluation and improvement proposal about tools and methods for effectively following up projects. Two main problems, representing the starting point of the work were identified in the management of projects:

1. The company with its tools lacks in an efficient and effective monitoring and controlling of projects; as a consequence, complete and reliable information on the progress of a project is often missing;

2. Inefficiencies in project monitoring and controlling, are sometimes caused by a not enough precise project planning.

As a consequence the purpose of the work was identified in the following aspects:

- One concerning the possible improvements on the current set of project monitoring tools, considering any possible inefficiency located into them;

- One regarding the introduction of innovative ideas which can effectively bring an advantage both in the project planning and follow up.

After the identification of the main problems and needs of the company the focus shifted in a proposal aiming at improving the way in which current projects are managed. The improvement proposal consists of three parts: the first two can be considered independently, while the third is an attempt to integrate the first and the second in a coherent unique proposal. The three parts are:

- The Anatomy Concept;

- The On Line Project Monitoring System;

- The integration of the two proposals.

\section{THE ANATOMY CONCEPT}

\subsection{What is New with the Anatomy Concept Approach: Focusing on the Product}

Most of the literature which can be found in books is mainly focused on projects and how they can be managed effectively and efficiently in order to deliver the product on time and keeping costs as low as possible. (Linn C. Stuckenbruck, 1981). There is a complete set of tools presented and some of them have already been introduced in the previous chapters. They all are an attempt to utilise in the best way all the resources which are available, and aid the Project Manager to understand where to focus in order to respect the plans and shorten the discrepancies generated from deviations occurring during the running project. 
The Gantt chart for example can be either a general or a detailed plan for every project member regarding the activities to be undertaken daily or weekly. But it can even be a schedule which shows the resource plan and how the resources vary while the project is proceeding. From this chart, a Project Manager knows exactly how to move resources within a project: it means that a person can be moved to perform another activity as long as the planned activity is already completed.

Other information is taken from the PERT and CPM diagrams, which tell the PM where are the critical phases of the project and what are the delays that should be minimised in order to respect the final delivery to the customer. (Jack K. Meredith, Samuel J. Mantel, Jr. 1995), (Kemps, R.R. 1995). Moreover, the Earned Value concept shows if the task is under control or some corrective action is required. The net amount of money is plotted against time and by evaluating the position of the task on a graph, the PM knows if that task has a delay or is respecting the main requirements. Thus, the PM can require further analyses on it or shift the focus on other matters. All these tools are concentrating on projects, but none of them, or at least very few, pay much attention on the outcome of the project, that is the product. The Anatomy Concept considers a new way of planning and following up projects based on the description of the product from a functional point of view. Through the identification of the product functions, representing the outcome of a project from a customer perspective, the management of a project results more efficient and customer oriented.

\subsection{The Anatomy Concept}

The Anatomy Concept is a way of considering the product, that is the outcome of a project, in terms of functions or system abilities. (Jack Järkvik, 1994)

It basically consists of two charts:

- The Anatomical Chart, a way of describing the product, that is the outcome of a project, in terms of functions or system abilities;

- The Integration Driven Plan which is a method, based on the Anatomical Chart, for planning and following up a project.

The Anatomical Chart is a chart which shows the product in terms of functions ${ }^{1}$. In other words it is a description of the product from the user's perspective. Each chart is graphically defined by several boxes; each box represents a function of the system. Every box contains just one ability of the whole system, while the whole set of boxes represents the complete system in terms of what it must do while functioning. For example, a system for managing a cellular network must be able to present the network status, present the list of alarms received from the switches, logging the alarms and solving the problems related to the alarms themselves.

\footnotetext{
${ }^{1}$ Functions and system abilities are here intended to the same extent. Every function or system ability reproduces one system capacity of performing specific activities
} 


\section{The Anatomical Chart vs. the Product Structure}

There is a substantial difference between the Anatomical Chart and the Product Structure. The first, as mentioned above, is showing the abilities the system has to accomplish once functioning. The Product Structure is dealing more with the components as parts concurring to constitute the product itself. For a mobile phone, the different components can be the receiver, the transmitter, the numeric pad, the antenna and so on.

\section{Reporting Progress on the Anatomical Chart}

One of the best uses of the Anatomical Chart is as a chart of progress. The Anatomical Chart shows the order in which results need to be seen. Thus, it is even possible to show what has actually been completed (Jack Järkvik, 1994). On one chart it is possible to show the result of system design. On a second chart how far the design work has progressed, or rather exactly what has been delivered for integration. A third one can show how much functioning has been got in the integration work in the system environment.

A good way of reporting the progress is simply to colour the various boxes on the chart. It is possible to define a set of three different colours to include the main possible development states for a function. The possible states are:

- the function is not delivered yet, despite the plans required it to be already functioning;

- effort is still put on it considering what was declared in the plan schedule;

- the function is already delivered and is functioning.

For each one of the three different cases there is a colour, which expresses the status of the function:

- green stands for "completed on time";

- red which is for "delayed";

- yellow that means "still working on it".

The three different colours are displayed in the single boxes of the anatomical chart. It is very important that the meaning of each colour is very clear and understood by all. The progress chart can be put where all can see them and updated daily. The results shown on the anatomical chart must be clear and unequivocal; this means that the chart should be typically on the negative side: the situation is always at least as good as the chart shows. It can be very healthy to show a conservative picture of the current situation. It teaches people that they can believe what others say and, conversely, that others should be able to believe what you yourself say.

From the progress report based on the anatomical chart it is possible to draw specific conclusions on what the system/product can be used for at that moment. 
In the Company the Anatomical Chart has been defined for a strategic product and, as an example, is reported below:

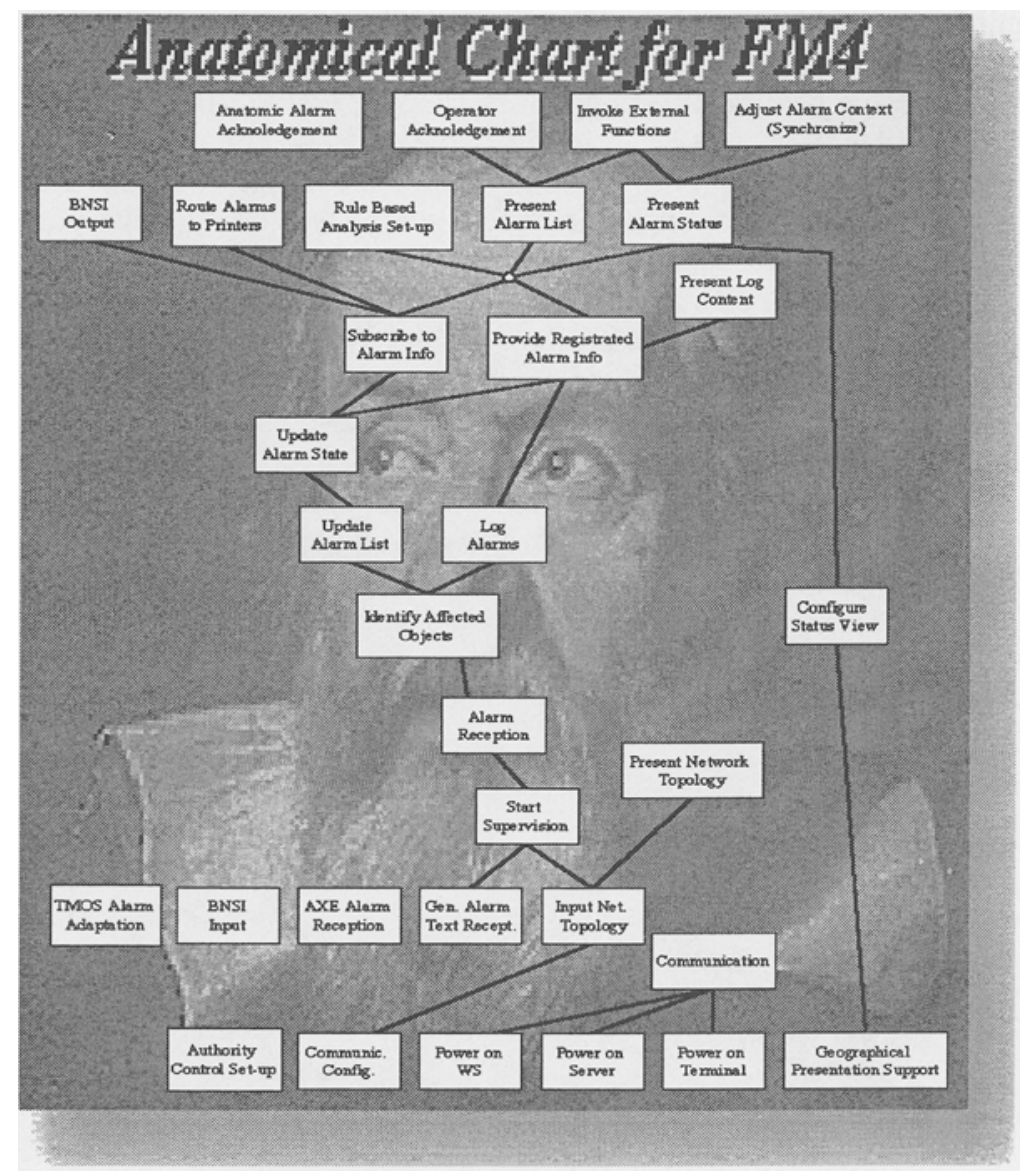

Figure 1 An example of the Anatomical Chart

A further step in the Anatomical chart is the definition of the Integration Driven Plan which represents the connection between the product and the project. The expression "Integration Driven" is used to indicate that the entire project is characterised by the order in and the time at which the various products and results need to be ready, being determined by when they are to be integrated. An advantage brought from the application of the Integration Driven Plan, is that what is considered here is product and the project together. The project dimension is included by reporting the time at which different functional deliveries of the product have to be made. 


\section{THE ON LINE PROJECT MONITORING SYSTEM (On Line PMS)}

In parallel to the Anatomy Concept Proposal it has been proposed the On Line Project Monitoring System which allows the Project Manager to have a continuous follow up of the progress of a project. The On Line PMS consists of a database where data concerning the project progress are continuously inserted and updated; when needed these data can be sorted from the database and used for monitoring and controlling the project itself. The purpose of the On Line PMS is to provide the Project Manager with a powerful and reliable tool in order to have precise and updated data for monitoring and controlling the project during its development.

The On Line PMS is "On Line" since it is supposed to work in the Intranet, an internal network, functioning on all the PCs and Work Stations in the company, where information is continuously exchanged among all the employees. The On Line PMS has been developed in order to become compatible with the internal Intranet. The data on the project progress are intended to be continuously inserted in the database, so updated information is always available.

It is possible to insert data in the database according to some product and project variables that identify relevant aspects of the project performance. The data are gathered and inserted during the project so the current performance can be compared with the planned performance and, as a consequence, corrective action can be taken.

Table1: Project Progress information needed by the Project Manager

$\begin{array}{cc}\text { Project variables: } & \text { Test cases } \\ \text { Costs } & \text { Product documents } \\ \text { Number of people } & \text { Requirements } \\ \text { Man-hours } & \text { Trouble reports } \\ \text { Project Documents } & \text { Functionality (\# of Functions) } \\ & \end{array}$

Project Variables are connected to the activities undertaken in the project in order to achieve the result of the project itself. The allocation of resources (in terms of costs, number of people and man-hours) in the different project activities, is necessary information that the Project Manager needs to constantly keep the project under control. Moreover the number of documents to be produced for the management of the entire project and for its phases needs to be known.

Product variables aim at identifying the key dimensions through which the progress of the product being developed will be defined. That is, in other words, to know the current status of the product and to know what is left to be done in order complete the final product as required by the customers. The five variables we identified (Test cases, Product Documents, Functionality, Requirements and Trouble Reports) are considered, by the majority of the employees we interviewed, as the main information by which it is possible to monitor continuously the actual development status of a product. 
What Is the Output of the On Line PMS - the Usefulness for the Project Manager

In each moment of the execution of the project the Project Manager and every other employee in the company can have "reading access" to the On Line PMS in order to sort the information related to product and project variables for a specific project. The information for each variable is given in terms of the actual performance compared to the planned performance. A graphical representation, intended as cumulative curves for the actual and planned performance respectively is provided:
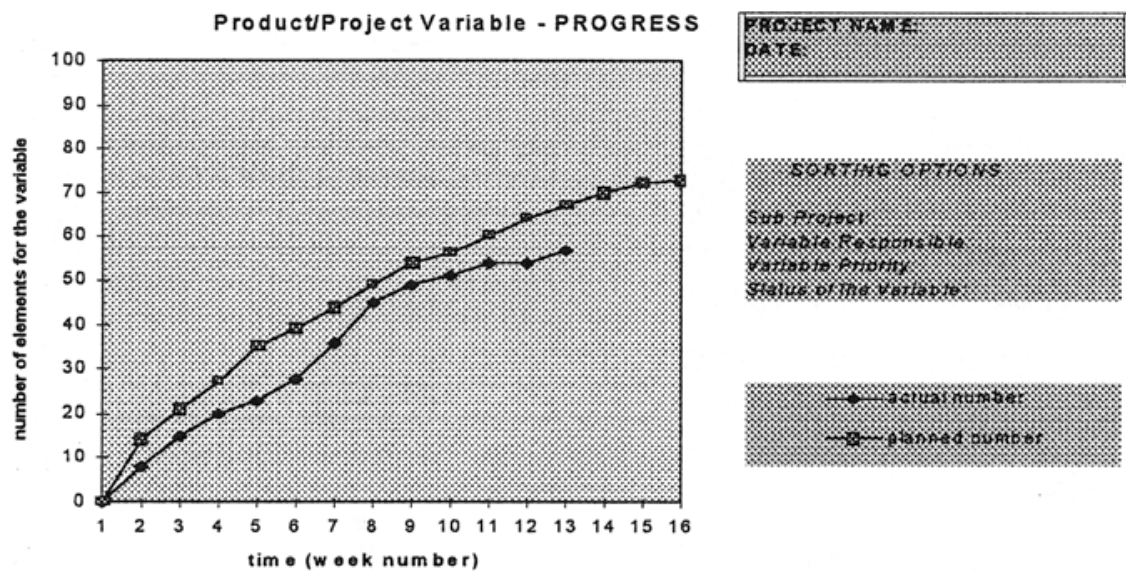

Figure 2 Presentation of the progress in the ON Line PMS

\section{THE INTEGRATION OF THE TWO SYSTEMS}

After presenting the Anatomy Concept proposal and the On Line Project Monitoring System proposal it has been evaluated why, how and to what extent the two proposals can be effectively integrated in order to have a complete and reliable measure in terms of the progress of the project. The integration is based on the project progress data coming from the On Line Project Monitoring System which are the input for the Integration Driven Plan where the progress of a project can be clearly and effectively drawn. The On Line PMS provides a measure of the progress of the project showing a number of graphs where the actual value of performance for some variables is compared with the planned value of the performance. The output is then, a number of curves by which the Project Manager and different Sub-Project Managers can check where in the project problems occur or resources are needed; then the necessary effective actions can be taken. Over the curves coming from the On Line PMS the Project Manager should have a way to 
control and report the status of the project that can be briefly and effectively presented.

A chart should be put on the wall and show to everyone (the project members but also other interested employees) the updated progress on the development of the project. Moreover, this chart can be used as a practical way for informing the Line Management, during the Steering meetings, and the customers about the development status of the project. The Integration Driven Plan is our answer to the need of having the progress of the project clearly showed in an A4 chart. The On Line PMS provides defined and clear data which can be "transferred" to the Integration Driven Plan in order to show the progress on it. In fact, by using the data coming from the On Line PMS it is possible to identify some criteria in order to define the progress colour for every box in the Integration Driven Plan.

\section{REFERENCES}

Linn C. Stuckenbruck, Ph.D. (1981). The Implementation of Project Management.

Project Management Institute. Addison-Wealwy Publishing Co., Inc.

Jack K. Meredith, Samuel J. Mantel, Jr. (1995). Project Management: A

Managerial Approach. John Wiley \& Sons, Int. p. 441-452

Jack Järkvik, Lars Kylberg (1994). A Bit Of Luck Helps Too. On Succeeding.p. 1018

Kemps, R.R. (1995) Fundamentals of Project Performance Measurement. San Diego Publishing Company.

Whitten, N. (1996) Managing Software Development Projects. New York: Wiley.

\section{Biography}

Enzo Gentili has been professor of Special Technologies at the University of Brescia, in Italy, since 1990. His main field of research regards Production Management, Modelling Tools and Techniques, Factories of the Future, Performance Management, Industrial Quality Management and Safety of Surgical Robots.

Postgraduate Claudio Nidasio has studied Industrial Engineering and Management at Brescia University (Italy). He has a Master of Science in Management of Production obtained in Chalmers University of Technology (Sweden).

Postgraduate Marco Varchetta has studied Industrial Engineering and Management at Brescia University (Italy). He has taken several management courses at Lulea University of Technology (Sweden). 